\title{
Myrtia, $\mathbf{n}^{\circ} 35$ (2020), 395-418
}

\section{Los Aphthonii Sophistae Progymnasmata de Bartolomé Alcázar: un manual de iniciación retórica del XVII}

\author{
[Bartolomé Alcázar's Aphthonii Sophistae Progymnasmata: a 17th-century \\ rhetorical manual for beginners] \\ María Elisa Cuyás de Torres* \\ Universidad de Las Palmas de Gran Canaria
}

\begin{abstract}
Resumen: $\quad$ En 1688 el jesuita Bartolomé Alcázar editó por tercera vez sus Aphthonii Progymnasmata. Este trabajo estudia el contenido, estructura y organización interna de su manual, comparando algunos de sus ejercicios con los de versiones anteriores, para comprobar si se trata de una traducción propia de la obra de Aftonio o una fusión de las de otros y determinar qué aporta al campo de la Retórica y de la Didáctica. Se han obtenido estos resultados: no es una traducción directa de Aftonio, sino la fusión de tres versiones humanísticas con comentarios, la de R. Lorich, B. Bravo y F. Pomey, cuyos contenidos esenciales Alcázar sintetiza y entremezcla, añadiendo algunos propios. No hay en ellos ningún precepto retórico nuevo, sino que su autor se ha centrado en realizar un manual práctico y asequible para los aspirantes de los estudios de Retórica que les facilite los conocimientos retóricos básicos para acceder a ellos sin problemas. Su aportación es puramente didáctica. Consigue un manual muy breve, y, sin embargo, claro y completo.
\end{abstract}

\begin{abstract}
In 1688 the Jesuit Bartolomé Alcázar published his Aphthonii Progymnasmata for the third time. This work studies the content, structure and internal organisation of his manual, comparing some of his exercises with those of previous versions, in order to (a) check whether it is his own translation of Aphtonius' work or a fusion of those of others, and (b) to determine the contribution of this work to the field of Rhetoric and Didactics. The results of this paper can be summarised as follows: it is not a direct translation of Aphtonius, but the fusion of three humanistic versions with commentaries, that of R. Lorich, B. Bravo and F. Pomey, whose essential contents Alcázar synthesises and intermingles, adding some of his own; there is no new rhetorical precept in them, but their author has focused on making a practical and accessible manual for aspirants to the studies of Rhetoric that will facilitate the basic rhetorical knowledge; his contribution is purely didactic. He manages to write a very brief, yet clear and complete manual.
\end{abstract}

Palabras

clave:

Retórica, Humanismo, progymnasmata, Bartolomé Alcázar

Keywords: Rhetoric, Humanism, progymnasmata, Bartolomé Alcázar

Recepción:

$27 / 03 / 2020$

Aceptación: $\quad 10 / 06 / 2020$

"Dirección para correspondencia: Departamento de Filología Hispánica, Clásica y de Estudios Árabes y Orientales. Universidad de Las Palmas de Gran Canaria. C/ Pérez del Toro, 1. 35003 Las Palmas de Gran Canaria (España). E-mail: mariaelisa.cuyas@ulpgc.es

Este trabajo se enmarca en el Proyecto de Investigación FFI2014-53859-P, financiado por el MINECO Gobierno de España. Forma parte de los estudios realizados en el Instituto Universitario de Análisis y Aplicaciones Textuales (IATEXT). 
El presente trabajo se centra en el estudio de los Progymnasmata de Aftonio de Bartolomé Alcázar ${ }^{1}$, jesuita murciano de la segunda mitad del XVII y primer cuarto del XVIII. Se estudiarán distintos aspectos: su estructura, su contenido, su organización interna y se compararán algunos de sus ejercicios con los de las versiones de otros autores anteriores, para comprobar si son fruto de una traducción directa de la obra de $\mathrm{Aftonio}^{2}$, según parece indicar el título, o una adaptación libre de la del rétor griego mezclada con ideas propias ${ }^{3}$, o son el resultado de varias traducciones anteriores ${ }^{4} \mathrm{y}$, en este caso, se determinará cuáles fueron sus fuentes, cómo las usó y veremos qué similitudes o diferencias presenta con ellas. Por último, se precisará cuáles son las aportaciones de este manual al campo de la Retórica y de la Didáctica, si las hubiere.

Hemos organizado nuestra exposición en los siguientes apartados: autor, obra, concretamente los Progymnasmata, fuentes, estructura y contenidos, terminaremos con las conclusiones que se desprenden del estudio.

${ }^{1}$ Se han publicado otros dos trabajos previos y complementarios sobre esta obra. Uno, en el que se da a conocer y estudia una edición perdida de la primera obra del autor, la Sylua selectorum tripartita ex probatissimis Latini sermonis authoribus collecta: Pars I Oratoria $\mathscr{E}^{\circ}$ Historica, Pars II Poetica, Pars III Didactica (1706). En ella también aparecen estos ejercicios. Solo se han identificado tres ejemplares no conocidos hasta entonces, uno bastante mutilado, precisamente en la parte que afecta a los Progymnasmata. Esta edición de 1706 sirvió de modelo a la de 1725, copia de la Universidad Complutense muy conocida por su digitalización (M.E. Cuyás de Torres, 2018a). El segundo estudio examina y compara todas las ediciones en que los Progymnasmata de B. Alcázar se dieron a conocer, ya que, al existir diferencias entre ellas, convenía analizar estas para determinar cuál era la mejor para estudiarlos (M.E. Cuyás de Torres, 2018b).

2 Algunas versiones en latín son, entre otras, la de Agrícola, publicada después de su muerte por Alardo (1532 y 1539), las de Cataneo (1507 y 1517), la de Bonfini (1538), la de Antonio Llull (1550, 1551, 1572), y la de Francisco Escobar (1558). Este, por falta de salud y posterior fallecimiento, solo comentó el ejercicio de la fábula. Sobre estas ediciones, véanse: para la de Agrícola de Alardo, M. D. García de Paso, 2015, pp. 1258-1261; sobre las dos ediciones de Cataneo, T. Arcos Pereira, 2017; sobre las ediciones de los Progymnasmata de Llull, M.E. Cuyás de Torres, 2016; y sobre el ejercicio de la fabula de Escobar, T. Arcos Pereira-M.E. Cuyás de Torres, 2008.

${ }^{3} \mathrm{La}$ primera adaptación libre de estas características es la de Pedro Moselano. Este adaptó la obra de Aftonio (1523) con la supresión de ejercicios y ejemplos e incorporación de otros, varios suyos, y añadió preceptiva propia. El resultado fue una obra nueva y original con gran repercusión en los humanistas posteriores, como demuestra M.D. García de Paso, 2018, pp. 165-173.

${ }^{4}$ Un ejemplo de fusión es la versión de R. Lorich, la Agricola partim, partim Catanaeo..., que mezcla Agrícola con Cataneo (1542 y 1546), estudios sobre esta edición en M.E. Cuyás de Torres, 2014, pp. 153-177 y M.D. García de Paso, 2018b, pp. 382-390. 


\section{1.- $\mathrm{El}$ autor}

Su autor, el humanista Bartolomé Alcázar (1648 -1721), perteneció a la Compañía de Jesús desde muy joven ${ }^{5}(1644)$ y ejerció en ella diversos cargos ${ }^{6}$ además de impartir clases ${ }^{7}$. En el año 1700 fue nombrado Cronista de la Societas Iesu de la provincia de Toledo, Historiae huius Prouinciae scriptor, cargo que desempeñó hasta su muerte. En el año 1713 es junto con J. Casani, también jesuita y Maestro de Matemáticas en el Colegio Imperial, miembro fundador de la Real Academia Española. Ambos compartieron Colegio y trabajaron en la elaboración de la primera edición del hoy llamado Diccionario de Autoridades de la RAE, cuyo primer volumen se editó en 1726, cinco años después de la muerte de Alcázar ${ }^{8}$.

${ }^{5}$ Ingresa en 1664 con 15 años, recibe Órdenes Sagradas en 1675 y hace profesión solemne de sus votos en 1682 (F. Sánchez Ruiz, 1947-1948, pp. 671-676).

${ }^{6}$ Prefecto de Letras Humanas en el Colegio Imperial de Madrid (1687-1692), Rector del Colegio de Cuenca (1692-1695) y Catedrático de Matemáticas en el Imperial de Madrid (1695-1700), M.E. Cuyás de Torres, 2018b, p. 247 y bibliografía allí citada. Los cálculos se han hecho por cursos escolares, pues las Órdenes religiosas dedicadas a la docencia efectúan habitualmente los traslados al finalizar los cursos académicos. Por eso, creemos que cuando su biógrafo Sánchez Ruiz dice que ha leído en el catálogo de su Orden que en 1693 “ya llevaba año y medio” en el Colegio de Cuenca (Sánchez Ruiz 676), el catálogo no se referiría a años naturales, sino al curso 1692-1693.

7 Según su biógrafo F. Sánchez Ruiz, en el que se basan todos los datos biográficos posteriores, Alcázar, cuando acabó en Madrid sus estudios de Filosofía, impartió Gramática en el Colegio de Oropesa (1667-1668) y de allí regresó a Murcia a estudiar Teología durante cuatro años (1668-1672) y a recuperar su salud algo mermada (mediocres vires). En el Colegio Imperial de Madrid enseñó Gramática dos cursos seguidos y, a partir de 1675, Retórica hasta el curso 1687-1688 en que fue nombrado Prefecto de Letras, cargo que conservó hasta su traslado a Cuenca en 1692 (F. Sánchez Ruiz, 1947-1948, pp. 672-678). A pesar de su trabajo encomiable y laborioso, a Sánchez Ruiz no le queda clara la afirmación de Alcázar de que “enseñó también Letras Humanas en Toledo” sin que haya más constancia (F. Sánchez Ruiz, 1947-1948, p. 673), sin advertir que el humanista comenzó su docencia en Oropesa, municipio de la provincia de Toledo, y que Alcázar mencionaría Toledo refiriéndose a Oropesa en sentido amplio.

${ }^{8}$ En la pág. X de la breve "Historia de la Real Academia Española”, tomo I, que forma parte de los estudios preliminares del Diccionario de la lengua castellana, hoy llamado Diccionario de Autoridades, figura una reseña de sus fundadores, que recoge que Alcázar falleció el 14 de enero de 1721. 


\section{2.- Obra}

El autor de los Progymnasmata escribió muchas obras de distintos géneros. Dentro de las históricas, la más importante es la Chrono-historia de la Compañía de Jesús en la provincia de Toledo (1710). También cultivó algunas hagiografías de jesuitas, como S. Ignacio y S. Francisco Javier, o la de S. Julián, segundo Obispo de Cuenca, que compuso estando destinado en el Colegio de esta ciudad (1691-1694); y otras de índole social o académica. Mejoró y editó, sin añadir su nombre (Sánchez Ruiz, 1947-1948, pp. 676 y 682), la adaptación de Nebrija del jesuita Juan Luis de la Cerda, Expositionem Syntaxeos atque Prosodiae Antonii Nebrissensis (1687). Es autor de varias obras didácticas, vinculadas con su docencia, que dio a conocer en forma de manuales ${ }^{9}$, y de traducciones o artículos en revistas extranjeras sobre astronomía, geografía o entomología $a^{10}$, materias a las que por obediencia tuvo que dedicar sus últimos años como docente.

\section{3.- Los Aphthonii Progymnasmata de Bartolomé Alcázar}

Los Aphthonii Sophistae Progymnasmata de Bartolomé Alcázar, objeto de este estudio, forman un pequeño manual que el autor debió de componer como base de sus clases de Retórica. Se publicó en cuatro ocasiones y siempre integrado en una obra más amplia ${ }^{11}$. En la primera de ellas, la Silua selectorum tripartita ex probatissimis latini Sermonis authoribus collecta: Pars

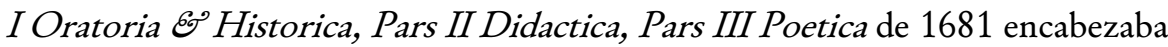
la Pars II o Didactica. La Pars II, concebida por su autor o tal vez por el editor como una obra independiente, se publicó casi simultáneamente el mismo 1681 como una "emisión formal" de la obra original ${ }^{12}$, con el nombre de De ratione dicendi. Opusculum triplex siue I. De Aphthonii sophistae progymnasmatis, II De conscribendis epistolis, III De rhetorica facultate libri quinque. En ella los Progymnasmata ocupan la primera posición. Años más tarde, en 1688, se

${ }^{9}$ La Silua selectorum tripartita... (1681a y 1706), el De ratione dicendi... (1681b y 1688), El perfecto latino en prosa y verso (1683), etc.,...

${ }^{10}$ Cf. una enumeración más detallada en M.E. Cuyás de Torres, 2018b, pp. 257-258 y, sobre todo, en F. Sánchez Ruiz, 1947-1948, pp. 681-687. También puede consultarse la nueva edición bilingüe De Ratione Dicendi de M. López-Muñoz, 2019.

${ }^{11}$ Véase un estudio completo de estas ediciones y sus contenidos en M.E. Cuyás de Torres, 2018a y 2018b.

${ }^{12}$ Cf. M.E. Cuyás de Torres 2018b: 256-257 y n. 37. 
reeditó esta Pars II independiente, corregida y aumentada, De ratione dicendi siue I Aphthonii sophistae progymnasmata, II De conscribendis epistolis, III De rhetorica facultate libri tres. En 1706 los Progymnasmata volvieron a aparecer publicados en la Pars III de una edición, probablemente ilegal, de la obra inicial completa ${ }^{13}$, que presenta importantes cambios respecto a la primera, la Sylua selectorum tripartita ex probatissimis Latini sermonis authoribus collecta: Pars I Oratoria $\mathcal{E}^{\circ}$ Historica, Pars II Poetica, Pars III Didactica, pero que, sin embargo, no recoge en su Didactica las mejoras y adiciones realizadas en la De ratione dicendi de 1688, salvo la corrección de algunas erratas sin importancia de los Progymnasmata, que no presuponen la consulta de la anterior de 1688 .

Para el estudio de la Silua selectorum tripartita ex probatissimis latini Sermonis authoribus collecta: Pars I Oratoria E० Historica, Pars II Didactica, Pars III Poetica, Mantuae Carpetanorum, typis et sumptibus Io. Garciae Infançonis (1681a), que citaremos en este artículo con sus siglas y el año de publicación, SST (1681a), se han utilizado los ejemplares impresos de la Biblioteca Nacional (BNE), de la Sede de Recoletos, signaturas 7/16520 y 2/35549. También se ha consultado la microforma de la BNAlcalá, DGMICRO/33257 y código de barras 11051500344 .

El estudio del De ratione dicendi. Opusculum triplex siue I. De Aphthonii sophistae progymnasmatis, II De conscribendis epistolis, III De rhetorica facultate libri quinque, Mantuae Carpetanorum, Typis $\sigma^{\circ}$ sumptibus Ioannis Garcia Infançonis (1681b), a partir de ahora citada por sus siglas y el año de publicación, $D R D(1681 \mathrm{~b})$, se ha hecho por el ejemplar de la BNE, Sede de Recoletos, signatura 3/33558, y por la microforma de la BNAlcalá, DGMICRO/10577, código de barras 1104058030.

El De ratione dicendi siue I Aphthonii sophistae progymnasmata, II De conscribendis epistolis, III De rhetorica facultate libri tres, Matriti, ex typographio Jo. Garciae Infançonis (1688), en adelante $D R D$ (1688), se ha estudiado por el ejemplar de la BNE, signatura 2/24255 y enlace digital: http://bdh-rd.bne.es/viewer.vm?id=0000089610. Se ha utilizado el ejemplar de la Sede de Recoletos, su microforma: RMICRO/28592 con código de barras 1001171628 y el texto digitalizado.

13 Véase un estudio sobre esta edición en M.E. Cuyás de Torres 2018a. 
La Sylua selectorum tripartita ex probatissimis Latini sermonis authoribus collecta: Pars I Oratoria $\mathscr{E}^{\circ}$ Historica, Pars II Poetica, Pars III Didactica, Mantuae Carpetanorum, Haeredes Antonii Roman (1706), para citas sucesivas $S S T$ (1706), se ha estudiado por los tres únicos ejemplares que se han identificado: el de la BNE, con signatura 3/42192; el de la Biblioteca Pública del Estado (BPE) de Ciudad Real, signatura F.A. 2368; y el de la BPE de Toledo, signatura F.A.16721. El primero de ellos está en muy mal estado y ha perdido la Pars III, en la que se encuentran los Progymnasmata.

Por el número de veces que se publicó la De ratione dicendi y las reformas que el autor introdujo en la de 1688, pensamos que fue una de sus obras predilectas y a la que dedicó más atención. De estas cuatro publicaciones de los Progymnasmata se ha escogido para estudiarlos la $D R D(1688)$, porque, según se ha probado en un trabajo previo (M.E. Cuyás de Torres, 2018b, pp. 277-281), esta es la edición que ofrece el mejor corpus, ya que presenta corregidas las erratas de sus predecesoras y ofrece el texto más completo de la versión de Aftonio de Alcázar, pues es la única que ha desarrollado el ejercicio de la narratio, de manera que en las otras el autor debe remitir para su estudio a la Rhetorica. También es la más adecuada por mostrar, a diferencia de las demás, ciertas alteraciones en la disposición interna del Progymnasma XII, que corresponde a la descriptio (DRD 1688, pp. 13-14 y M.E. Cuyás de Torres, 2018b, p. 269).

Un estudio completo de los Progymnasmata de Alcázar, como ya se ha señalado en nuestro trabajo sobre sus ediciones (M.E. Cuyás de Torres, 2018b, pp. 278-281), no se puede limitar solo a los ejercicios preliminares, sino que requiere contar también con los comentarios del propio humanista a propósito de su elaboración en la Carta al Lector de la edición, $D R D(1688)^{14}$; con los que incluye en la Carta al Lector que ofrece solo la $S S T(1681 \mathrm{a})^{15}$ entre las Partes Iy II; y con los que aparecen en la especie de prólogo que precede a la Pars II en las dos ediciones de 1681, pues lo expuesto en estos textos ayuda a identificar sus fuentes y explica, además, cómo ha realizado la parte a la que pertenecen los Progymnasmata y que es la que él considera más genuinamente suya.

${ }^{14} D R D(1688)$ ff. 6r-6v s.n. Comentario en M.E. Cuyás de Torres, 2018b, pp. 267$269 ; 276 ; 279$ у 281.

15 SST (1681a) f. r-v s.n. (M.E. Cuyás de Torres, 2018b, pp. 260-262; 276; 278-279 y 281). 
Los Aphthonii Sophistae Progymnamata de Bartolomé Alcázar, según se ha dicho al inicio de su estudio, son un manual de pequeñas proporciones que en la edición corregida y aumentada, $D R D(1688)$, consta de unas dieciséis páginas no completas ${ }^{16}$, porque la última de estas solo ocupa la mitad. Forman parte del De ratione dicendi, que se corresponde con la Pars II de la obra original, la SST (1681a), y con la Pars III de la SST (1706). El De ratione dicendi es un tratado que el autor dedicó a la docencia de la Retórica. De ahí que en esa edición inicial, según se ha indicado en la introducción a la obra ${ }^{17}$, el autor haya designado a esta Pars II Didactica, denominación que evidencia claramente que fue compuesta para la enseñanza.

En la portada de la edición de 1688 Alcázar añade como nombre alternativo de la $D R D$ (1688) los títulos de los tres manuales que componen esta obra tripartita, [...] opusculum triplex: siue I. Aphthoni Sophistae Progymnasmata, objeto del presente estudio, II. De conscribendis epistolis, un tratado epistolar, y III. De rhetorica facultate, libri tres, un manual de Retórica, con el que finaliza.

El contenido de la obra parece revelar una enseñanza que se adapta al plan de estudios habitual en la Compañía de Jesús, regulado por la Ratio Studiorum ${ }^{18}$, con tres niveles progresivos. En ellos los Progymnasmata constituirían el nivel inicial de los estudios retóricos y proporcionarían a los alumnos los fundamentos básicos o primeros peldaños por los que podrían acceder a los conocimientos más sólidos de la preceptiva retórica, el tratado epistolar representaría el grado intermedio y la Rhetorica el último ${ }^{19}$.

${ }^{16}$ En las otras ediciones tiene aún menor extensión: en las $S S T$ y $D R D$ de $1681 \mathrm{~b}$ (2r8r) consta de siete folios y medio del $2 \mathrm{r}$ al $8 \mathrm{r}$ que equivalen a doce páginas y media y en la SST 1706 (pp. 1-13) con la misma longitud o sea una página menos en todas que en la de 1688. El incremento de páginas en la de 1688 se debe al desarrollo en esta de la narratio, omitido en las anteriores, y al tamaño de la letra.

17 Ver supra, párrafo inicial de este apdo. 3.

18 Ratio atque Institutio studiorum Societatis Iesu, 1599. Sobre los estudios en la Compañía de Jesús pueden consultarse E. Gil, 1992 y T. Arcos Pereira (en prensa), apdos. 1.c. y 2 .

${ }^{19}$ Ya Bravo afirmaba en el proemio a sus Progymnasmata que estos, según Quintiliano, debían ser como el peldaño más bajo para subir al más alto de la Retórica: ex hoc enim quasi gradu ad altiora illa ac sublimiora facilius ascendent (Bravo, 1591, f. 58v). 
3.1. ¿Versión de Alcázar o reutilización de las traducciones de otros humanistas? En la propia portada, tras los nombres de los manuales que integran la obra, Alcázar agrega como reclamo publicitario ${ }^{20}$ una breve síntesis de la misma, muy similar a los resúmenes que figuran hoy en los artículos de las revistas científicas: Opus ex Optimis Rhetoribus collectum $\mathcal{F}^{\circ}$ in breuissimam formam dilucidamque digestum. ( $D R D$ 1688, f. 1r, s.n.). Con esta indicación el autor, ya antes de su inicio, advierte que en su obra no se va a encontrar una traducción directa de los ejercicios de Aftonio hecha por él ni tampoco una preceptiva original de la teoría epistolar y retórica, sino una reelaboración selectiva de los mejores rétores en forma breuissima dilucidaque. En resumen, Alcázar notifica al lector, ya desde la portada, que su obra no es original, sino una selección muy breve y clara de las mejores retóricas anteriores. En efecto, si se cotejan estos Progymnasmata con los de Aftonio ${ }^{21}$, se ve que no son una traducción del texto griego, sino una amalgama de las teorías de los humanistas que han precedido a Alcázar, muchos de ellos también jesuitas. Por lo tanto, uno de los objetivos fundamentales de este estudio será el averiguar qué fuentes ha usado el autor en ellos y cómo las ha unificado para conseguir esta nueva versión del rétor griego.

\subsection{Las fuentes y su uso}

Alcázar nos habla de sus fuentes en la especie de segundo prólogo que precede a la Didactica en las publicaciones de 1681, las SST (1681a, f. 1v) y $D R D(1681 b, f .1 v)$. En él, después de explicar, con palabras de Justo Lipsio y Plinio el Viejo, cómo ha compuesto los tres manuales que la forman, da una lista de nueve autores que le han proporcionado el material para su realización (M.E. Cuyás, 2018b, pp. 262-263). En la $D R D(1688)$ vuelve a referirse a sus fuentes en una Carta al Lector, ff. 6r-6v, que sustituye al citado prólogo. En esta Carta enumera once autores de Retórica, a los que califica de Celebriorum Artis Magistrorum y de quienes dice que ha tomado preceptos y ejemplos (praecepta exemplaque deprompsì). De ellos, Aristóteles, Cicerón, Quintiliano,

20 Estos reclamos publicitarios, bastante habituales en las portadas de las ediciones humanísticas, debían de sugerírselo a los autores probablemente los editores o añadirlos motu proprio. Este figuraba ya en la SST(1681a, 1r, s.n.) en la portada interior de la Pars $I I$ y en la única portada de la $D R D(1681 \mathrm{~b})$.

${ }^{21}$ Se han comparado con el texto griego de Aftonio, establecido y traducido por $\mathrm{M}$. Patillon: Corpus Rhetoricum..., 2008, pp. 112-162. También se puede consultar la traducción española de M. D. Reche Martínez, 1991, pp. 207-268. 
Aftonio, Caussin, Pelleterio y Macrobio no aparecían en las ediciones anteriores; pero algunos, como Cipriano, Vossio, Bravo y Pomey, ya estaban recogidos en ellas y deja implícitos en la expresión aliorumque uoluminibus... perlectis (DRD 1688, f. 6r) a otros, cuya identidad se revela al leer la obra y reconocer sus textos. Esta Carta se centra en explicar las razones que le han impulsado a mezclar tantas preceptivas y ejemplos, alegando como causas fundamentales la importancia de los autores escogidos y la dificultad de sus alumnos no solo pecuniaria para adquirir tantas obras, sino también de falta de tiempo para poder leerlas todas, así como su carencia de formación para discernir lo mejor de cada una: quorum opportunitate necessitati Scholasticorum, concisione egestati et tempori, claritate intelligentiae prouiderem $(D R D 1688, \mathrm{f} .6 \mathrm{r})^{22}$.

De los autores mencionados por Alcázar como fuentes de su Didactica en los Progymnasmata únicamente se detectan ${ }^{23}$ tres de ellos: Marco Tulio Cicerón, Bartolomé Bravo (1554) y Francisco Pomey (1618-1673). Los dos últimos, jesuitas como él, son fuentes directas. De Bravo utiliza tanto el De conscribendis epistolis [...], Progymnasmata siue Praeexercitationes Oratoriae..., $1591^{24}$, como su De arte oratoria..., $1596^{25}$. En algunos ejercicios, como en el De legislatione ( $D R D 1688$, p. 16), acude a la segunda obra posiblemente, porque en el tratado anterior no se aborda, pero en otros también recurre a ella sin que se den estas circunstancias. De Pomey se sirve del Candidatus Rhetoricae, seu Aphthonii Progymnasmata in meliorem formam usumque redacta, Lugduni, apud Antonium Molin,166126. Usa con más frecuencia su obra que las de Bravo, porque es más claro en su exposición. De Cicerón solo recoge un pequeño fragmento del De legibus (leg.1.18.10) en el último progymnasma (DRD 1688, p. 16), pero el Arpinate no es fuente directa, sino indirecta, porque el texto citado y su frase parentética: (inquit Cicero) ${ }^{27}$ han

${ }^{22}$ Cf. M.E. Cuyás, 2018b, pp. 267-269.

${ }^{23}$ En este estudio solo se tratarán las fuentes de los Progymnasmata.

${ }^{24}$ Hemos consultado el ejemplar de la Biblioteca de la Universidad de Sevilla con signatura A Res. 28/7/26.

${ }^{25} \mathrm{La}$ consulta de este ejemplar se ha realizado a través de la Biblioteca Virtual Larramendi con enlace: <http://www.larramendi.es/es/consulta/registro.do?id=27710> [25/02/2020].

26 Se ha consultado el ejemplar de la Bayerische Staatsbibliothek (BSB) <urn:nbn:de:bvb:12-bsb10574438-6> [25/02/2020].

${ }^{27}$ Reinhardus Lorichius Hadamarius (ca.1500-1564), pastor protestante y profesor de Retórica en la Universidad de Marburgo, publicó el más famoso comentario de los 
sido tomadas de Bravo (1596, ff. 218r-v: ut diffinit Cicero). Aportamos la definición de ley de Cicerón, Lorich y Bravo para que se puedan constatar las similitudes ${ }^{28}$ existentes:

- Lex est ratio summa, insita in natura, quae iubet ea, quae facienda sunt, prohibetque contraria (CIC. leg. 1.18.10).

- Lex, inquit, est ratio summa insita a natura, quae iubet ea quae facienda sunt, prohibit contraria (Lorich, 1670, p. 354).

- Legislatio, siue legis inductio est oratio, qua legem aliquam uel comprobamus, ac reprehendimus. Est autem lex, ut diffinit Cicero, ratio summa, insita a natura, quae iubet ea, quae facienda sunt, prohibetque contraria (Bravo, 1596, ff. 218r-v).

- Legislatio est oratio, qua legem aliquam uel approbamus, uel reprehendimus. Est autem Lex (inquit Cicero) ratio summa, insita a natura, quae iubet ea, quae facienda sunt, prohibetque contraria. (Alcázar, 1688, p. 16).

Es evidente, al cotejar los pasajes anteriores, que Alcázar utiliza a Cicerón no directamente, sino a través de Bravo (1596, ff. 218v), y que sustituye algunos términos por otros más usuales: comprobamus > approbamus; uel... ac > uel... uel; diffinit > inquit ${ }^{29}$. Simplifica mucho el texto: Legislatio, siue legis inductio est oratio $>$ Legislatio est oratio; omite lo que no le parece necesario: siue legis inductio > om., aunque conserva lo esencial de la definición: Est autem lex, ut diffinit Cicero, ratio summa, insita a natura, quae iubet ea, quae facienda sunt, prohibetque contraria $>$ Est autem Lex (inquit Cicero) ratio summa, insita a natura, quae iubet ea, quae facienda.

Progymnasmata de Aftonio. Por ser protestante, se expurgaron muchos de sus escritos. En sus scholia cita el mismo texto de Cicerón: Lex, inquit, est... (Lorich, 1670, p. 354; se ha consultado el volumen de la Biblioteca Nacional de España con signatura 3/57825). Lorich también es fuente de Bravo y Alcázar ha combinado los textos de ambos humanistas. Se utiliza la edición de Lorich de Vesalia (1670) por no estar expurgada y ser copia fiel de su tercera edición de Francfort de 1546, la más completa (M.E. Cuyás de Torres, 2014, pp. 153-154).

${ }^{28}$ En estos pasajes se destacan en redonda los términos que Alcázar cambia o descarta, en cursiva los que toma, aunque altere su ubicación.

${ }^{29}$ Aquí prefiere sustituir diffinit del texto de Bravo por el más común inquit empleado por Lorich. 
Una cuarta fuente no citada, pero sí muy presente en todo el manual, es el humanista alemán Reinhard Lorich. Alcázar en su Prooemium copia casi íntegramente con ligeras divergencias los scholia de Lorich a la fabula y luego recurre reiteradamente a él en los progymnasmata. Unas veces utiliza su edición Agricola partim, partim Catanaeo... y otras los escolios para complementar o explicar ciertos aspectos teóricos o definiciones ausentes en sus otras dos fuentes, Bravo y Pomey, o que no le satisfacen, porque el texto de Lorich aporta más claridad a sus explicaciones teóricas, además, el alemán es el autor preferido por los jesuitas como base de sus obras.

Alcázar aprovecha de sus fuentes la traducción de la parte teórica y de Lorich, además, sus escolios. De los ejemplos que Aftonio añade a los progymnasmata como modelos prácticos del ejercicio solo utiliza alguna explicación, guías de lectura y anotaciones marginales, especialmente los capita amplificationis o

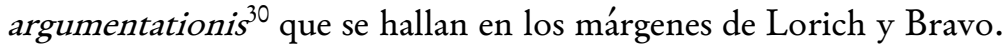

La utilización de las fuentes en los Progymnasmata no es uniforme. En algunos pasajes solo ha tomado como modelo a un autor: en el Prooemium, a Lorich, y en el De legislatione, a Bravo. Lo habitual, sin embargo, es que en cada ejercicio recurra a más de una fuente, aunque predomine una de ellas, pero no de forma exclusiva, sino entremezclada con las otras y glosada con explicaciones sintetizadas de Lorich o de Pomey. En los Progymnasmata Alcázar entrecruza las tres fuentes para lograr un manual más breve, propiciador del aprendizaje y útil para sus alumnos, guiado únicamente por la claridad del contenido. Por eso, su labor, como él mismo explica, es muy similar a la que realizan las abejas que liban de diferentes flores: Lapides et ligna ab aliis accipio [...] ex alienis libamus, ut Apes (SST 1681a y DRD 1681b, f. 1v). El jesuita reorganiza teorías ajenas y las dota de una nueva estructura. Con estos preámbulos deja claro que en la obra va a haber más de una fuente y que no siempre estarán nítidamente separadas, como podrá verse a continuación en estos pasajes del ejercicio de la fabula, tomados básicamente de Pomey, pero en el que subyace de forma clara Lorich:

- Est autem Fabula sermo falsus, ueritatem effingens. (Lorich, 1670, p.7).

- Quid est Fabula? Est sermo falsus, ueritatem effingens, hoc est; Falsa quidem et ficta narratio est; at sub fabuloso uerborum cortice, ueritas semper latet aliqua et utilis sensus (Pomey, 1661, 243-244).

30 Sobre los capitula finalia o argumentos y la descripción que hace de los mismos PRISC. rhet., 6 (M.E. Cuyás de Torres, 2014, p. 167, n. 67). 
- Fabula est sermo falsus ueritatis speciem effingens. Sub cuius commentitio uerborum cortice ueritas semper aliqua, et utilis sensus delitescit. Vnde, ficta quidem eius narratio est, sed uera eius significatio (Alcázar, 1688, p. 1).

En estos pasajes usados como ejemplo se observa que, unas veces, copia su modelo casi ad pedem litterae con escasas divergencias: Fabula? Est sermo falsus, ueritatem effingens $>$ Fabula est sermo falsus ueritatis speciem effingens; otras, parafrasea y sustituye algunos términos específicos por expresiones más coloquiales y asequibles a los jóvenes: sub fabuloso uerborum cortice, ueritas semper... aliqua > sub cuius commentitio uerborum cortice ueritas semper aliqua; con frecuencia, amplía los verbos con perífrasis, prefijos y sufijos: latet $>$ delitescit, e incorpora a las frases palabras implícitas o complementos que aclaran más el texto: ueritatem > ueritatis speciem; o bien altera la ubicación de los términos: semper latet... sensus $>$ semper... sensus delitescit. Normalmente, prefiere resumir la teoría y suprimir lo innecesario: hoc est; Falsa quidem et ficta narratio est $>$ Vnde, ficta quidem eius narratio est, si bien, en ocasiones, inserta explicaciones: sed uera eius significatio, que facilitan la comprensión de los contenidos. No es infrecuente que acuda a todos estos recursos en un mismo párrafo, como en este texto de la fabula. Al leer el manual y cotejarlo con los de otros humanistas, se percibe que Alcázar, como bien indica él mismo, ha seleccionado de aquí y de allí preceptivas, como las abejas con las flores para obtener una obra muy breve y fácil de entender para los que pretenden iniciarse en los estudios de Retórica.

\section{4.- Estructura y contenido de los Aphthonii sophistae Progymnasmata}

La versión de los Aphthonii Progymnasmata de Alcázar consta de un breve Prooemium, seguido de los catorce ejercicios de Aftonio, dispuestos en el mismo orden que en la obra del rétor griego: fabula, narratio, chria, sententia, refutatio, confirmatio, locus communis, laus, uituperatio, comparatio, ethopoeia, descriptio, thesis y legislatio.

\subsection{Prooemium}

El Prooemium de quince líneas (1688, p. 1), aunque de escasa extensión, es proporcional al conjunto del manual. En él el autor toma como modelo el inicio de los scholia de Reinhard Lorich al ejercicio de la fábula (Lorich, 1670, p. 8), en su Aphthonii Progymnasmata, a Rodolpho Agricola partim, partim a Johanne Maria Catanaeo. Alcázar lo inicia con una breve 
definición de los progymnasmata ${ }^{31}$ : 'Ea sunt minora dicendi praeludia' ( $D R D$, 1688 , p. 1), muy cercana a la del humanista alemán: Sunt autem opera minora (Lorich, 1670, p. 8) ${ }^{32}$. En el Prooemium el jesuita reutiliza los scholia de Lorich, como demuestran las palabras con las que precisa que estos ejercicios deben preceder a los estudios de Retórica y cuáles han de ser sus destinatarios: 'quibus diu multumque uersatos oportet esse adolescentes prius quam accedant ad iustam orationem pertractandam' (DRD 1688, p. 1), pues son una paráfrasis de los escolios de Lorich: adolescentes in minoribus dicendi facultatis operibus ante exercendos esse quam ad magnum illud Declamationum opus perducantur (Lorich 1670, p. 8). Antes de enumerar los ejercicios, copia casi ad pedem litterae: 'Quae quidem tribus hisce uersibus comprehenduntur' (DRD 1688, $\mathrm{p}$. 1), la introducción a ellos de Lorich, Quae ut pueri memoria facilius tenere possint, uersiculis hisce tribus sum complexus (Lorich 1670 p. 8) y repite los versos mnemotécnicos que facilitan el aprendizaje del nombre de los ejercicios:

Fabula, narratur, chria, sententia, futat,

Confirmat, locus haud priuus, laus, crimina, confert,

Fictio, descriptum, thesis, ac inductio legum

(DRD 1688, p.1; Lorich 1670, p.8).

Difiere de Lorich en que este enumera los ejercicios antes de los versos y Alcázar a continuación y precedidos del número que les corresponde en el orden de los progymnasmata:

Hoc est: 1. Fabula. 2. Narratio. 3. Chria. 4. Sententia. 5. Confutatio ${ }^{33}$.

6. Confirmatio. 7. Locus communis. 8. Laudatio. 9. Vituperatio. 10. Comparatio.

11. Ethopoeia. 12. Descriptio. 13. Thesis. 14. Legislatio

(DRD 1688, p.1).

${ }^{31}$ A partir de ahora, cuando recojamos pasajes de Alcázar, mantendremos la grafía de su edición. Usa cursiva para destacar términos o indicar que cita textualmente a su modelo y separarlo de sus explicaciones en redonda; también emplea mayúsculas para resaltar determinados vocablos: Orationem. Estos cambios de grafía son habituales en casi todos los textos humanísticos, especialmente en los destinados a uso escolar y, sobre todo, cuando los comentarios no se hallan separados del contenido teórico.

32 Sobre la influencia de Moselano en Lorich, precisamente en esta introducción a sus escolios, cf. M.D. García de Paso, 2018a, p. 174.

33 Alcázar en esta enumeración del Prooemium sigue tan fielmente a Lorich que para referirse a este ejercicio emplea el término Confutatio y lo conserva en la asignación de los progymnasmata a los tria genera (DRD 1688, p. 1). Sin embargo, al desarrollarlo (DRD 1688, pp. 6-7), utiliza el vocablo refutatio y el capítulo V que le dedica lleva como epígrafe en letras capitales DE REFVTATIONE. El término confutatio es el mismo que le da Moselano de quien lo tomaría Lorich, cf. M.D. García de Paso, 2018a, p. 174. 
Concluye el Prooemium, como Lorich, con la asignación de los ejercicios a los tres géneros, si bien presenta pequeñas divergencias con él en la vinculación de estos a los tria genera causarum. Lorich ${ }^{34}$ solo asigna doce ejercicios, pues no atribuye a ningún género la legislatio y la descriptio, mientras que Álcazar vincula los catorce ejercicios a los géneros, la legislatio al deliberativo y la descriptio al judicial y, por otro lado, adjudica la narratio también al género judicial frente a Lorich que la adscribe al deliberativo.

Cuadro comparativo de distribución de los Progymnasmata en los genera causarum

\begin{tabular}{llllll}
\hline $\begin{array}{c}\text { Genera } \\
\text { causarum }\end{array}$ & Deliberativo & \multicolumn{2}{l}{ Demostrativo } & & Judicial \\
\hline \multirow{4}{*}{ Lorich } & 1. Fabula & 8. Laus & 5. & Confutatio \\
& 2. Narratio & 9. Vituperatio & 6. & Confirmatio \\
& 3. Chria & 10. Comparatio & 7. & Locus communis \\
& 4. Sententia & 11. Imitatio/Ethopoeia & & \\
& 13. Thesis & & & \\
\hline \multirow{4}{*}{ Alcázar } & 1. Fabula & 8. Laudatio & 2. Narratio \\
& 3. Chria & 9. Vituperatio & 5. Confutatio \\
& 4. Sententia & 10. Comparatio & 6. Confirmatio \\
& 13. Thesis & 11. Ethopoeia & 7. Locus communis \\
& 14. Legislatio & & & 12. Descriptio \\
\hline
\end{tabular}

Posiblemente Alcázar ha elegido el proemio de Lorich como modelo, porque le permite en unas breves líneas presentar a los destinatarios qué son los progymnasmata y cuántos, cómo se los denomina, por qué deben estudiarlos y para qué les van a servir. De esta forma involucra más al alumno y consigue que sea un partícipe activo.

\subsection{Progymnasmata}

La versión de los Progymnasmata de Alcázar destaca por la reducida extensión de sus ejercicios ${ }^{35}$ : Tres de ellos, la confirmatio, la uituperatio y la legislatio, ocupan media página, le siguen en extensión la fabula, la chria y la

${ }^{34}$ Cf. la observación que hace M.D. García de Paso, 2018b p. 386, n. 30 y p. 387 sobre la asignación de los ejercicios a los tria genera en Lorich. Se ha utilizado un cuadro comparativo similar al suyo.

${ }^{35} \mathrm{La}$ extensión de los ejercicios se ha calculado por la real que abarcan con sus líneas y no por la numeración de las páginas. 
thesis con casi una página y media y los más largos son el locus communis y la ethopoeia con casi dos. Es evidente que el autor se ha propuesto como meta la máxima brevedad, a pesar de haber seleccionado en su obra lo mejor de la preceptiva retórica anterior, y que ha hecho realidad el dicho de Séneca de que es propio del gran artífice abarcar 'Totum in Exiguo', como afirma en la Carta al lector de esta edición (DRD 1688, 6r, s. n.).

Alcázar, como en el De conscribendis epistolis ${ }^{36}$, organiza de forma sistemática y obedeciendo a propósitos didácticos la estructura interna de sus Progymnasmata y así respeta en todos el mismo esquema descriptivo con adición de algún rasgo pertinente más, si la aportación de este dato favorece la comprensión de los alumnos o el desarrollo del ejercicio. La reiteración de estructuras similares es un método pedagógico que facilita el aprendizaje. Sigue, como Aftonio, este orden: definición, tipología o clases y puntos de argumentación de desarrollo o capita amplificationis (Aphth. Prog.). En el locus communis, cambiado de lugar, como Pomey, su modelo principal en este progymnasma, aunque también hay partes de Lorich, enumera los capita dilatandi y capita aduersus reum e indica que estos últimos son los que deben emplear los jueces (DRD 1688, pp. 8-9). En algunos ejercicios, por ejemplo la chria o el locus communis, como Aftonio, añade su etimología detrás de la definición (Prog. 3.1 y 7.1. Corp. Rhet., 2008, pp. 114 y 126-127): 'Dicta autem est Chria a uerbo Graeco, quod usum significat' (DRD 1688, p. 4) ) $^{37} \mathrm{y}$ 'Dicitur autem Communis, quod omnibus,... participes sunt communiter attribui possit' (DRD 1688, p. 8$)^{38}$, porque cree que en ambos casos la adición de este rasgo facilitará su comprensión y es pertinente. En la fabula, como Aftonio, proporciona la denominación de la moraleja según su posición (Prog. 1.3 Corp. Rhet., 2008, p. 113): 'ante Fabulae narrationem Praefabulatio uel Promythion; si post absolutam narrationem Fabulae, dicitur Affabulatio uel

36 Véase M.E. Cuyás de Torres, 2018b, p. 269.

37 En este tercer ejercicio, aunque utiliza mucho a Lorich, la fuente principal es el Candidatus Rhetoricae de F. Pomey y, de acuerdo con sus consejos, lo denomina chria y no chreia, como aparece en el texto de Lorich: Chreia est... (Lorich, 1670, p. 45). Explica Pomey que Chria dici debet non Chreia ut uulgus imperitorum, ut Gallice quam latine loquitur. [...] diptongus latinitate [...] exprimitur i longum (Pomey, 1661, p. 301).

38 También en esta definición del locus communis resume a Pomey: Quod communiter iis omnibus attribuit possit, qui eiusdem laudis aut uitii participes cum illo sunt... (Pomey, 1661, p. 406). 
Epimythion (DRD 1688, p. 2). Solo en la narratio, como en Aftonio, se enumeran las uirtutes (Prog. 2.4 Corp. Rhet., 2008, p. 114): 'Virtutes eius sunt quatuor. 1. Claritas, seu Perspicuitas; 2. Breuitas; 3. Probabilitas, seu uerisimilitudo; 4. Suauitas et electorum uerborum proprietas' (DRD 1688, pp. 3-4), aunque remite al libro II, capítulo 2 de su Rhetorica para la aclaración de cada una de ellas: 'Super quibus uide inferius Rhetor. lib.2.cap.2' (DRD 1688, p. 4). Utiliza la cursiva en las definiciones, ya sea para indicar con ello que han sido recogidas textualmente o para resaltar su importancia; en los puntos de argumentación solo la usa, si los explica. En la definición de tres progymnasmata, chria (p. 4), ethopoeia (p. 12) y thesis (p. 14) intercala en letra redonda conjunciones disyuntivas uelo seu para unir aclaraciones a la definición inicial.

En el progymnasma XII, De descriptione ${ }^{39}$, Alcázar toma como modelo único a Lorich, resume su versión Agricola partim, partim Catanaeo y la mezcla con sus scholia, que en la obra del humanista alemán alcanzan un total de doce páginas (Lorich 1670, pp. 305-316). Así logra un progymnasma claro y mucho más breve de una sola página ${ }^{40}$. La estructura es similar a la de los otros ejercicios: definición, uso y clasificación. En la edición de 1668 Alcázar innova en este ejercicio al reagrupar la clasificación de sus otras ediciones, de acuerdo con un nuevo criterio alfabético-semántico más lógico y fácil para los alumnos, así coloca primero todos los nombres, cuyo segundo elemento es - GRAPHIA y, a continuación, todos los que el segundo componente es -THESIA. Al final, para que los alumnos no se pierdan en medio de tantas denominaciones, añade una breve síntesis, tomada de la versión de Lorich ${ }^{41}$, en

${ }^{39}$ Hermógenes consideraba que este ejercicio no debía tener carácter independiente, porque ya se había tratado en otros anteriores, como la fábula, el relato, el lugar común y el encomio, aunque él lo incluyó en sus ejercicios preliminares para evitar ser acusado de negligencia (Hermog. Prog. 10.7 en Corp. Rhet..., 2008, p. 203). También Pomey omite este ejercicio y la ethopoeia en su obra el Candidatus Rhetoricae, con la justificación de que los dos tienen prácticamente los mismos preceptos que la narración: Nec de DESCRIPTIONE, quicquam ex Aphthonio subiicio, non tantum quia nullis praeceptis continetur, eadem ferme Descriptioni quae Narrationi conveniunt, et cum Ethopoeia eadem prorsus est (Pomey, 1661, p. 408).

${ }^{40}$ Solo ocupa las líneas finales de la página 13 y gran parte de la página 14.

${ }^{41}$ Utilizamos redonda en el texto de Lorich para destacar las diferencias con el de Alcázar. En la descripción de personas las diferencias son más acusadas. En cambio, en las descripciones de res, tempora y loca solo presenta ligeras modificaciones. 
la que los objetos de la descripción aparecen en cursiva para resaltarlos: Describentes uero personas a summis ad ima usque ire oportebit, id est, a capite ad pedes. (Lorich, 1670, p. 305) > Persona describitur a totius corporis habitu, et a locis laudis, uel uituperationis uel comparationis (DRD 1688, p. 14). Res uero $a b$ antecedentibus, et eis quae insunt, ipsis, quaeque ex ipsis solent prouenire. (Lorich, 1670, p. 306) > Res ab antecedentibus et quae in ipsis rebus insunt, quaeque ex ipsis solent prouenire. V.g. Vrbis expugnatio. (DRD, 1688, p. 14). Tempora uero et loca ex ea, quae ipsis continentur (Lorich, 1670, p. 306) > Loca, $\mathfrak{G}^{0}$ Tempora, a rebus, quae in ipsis uel continentur, uel fiunt. (DRD, 1668, p. 14).

El número de ejemplos en los progymasmata es muy variable: en cinco, fabula, narratio, laus, uituperatio y legislatio no aparece ninguno. En los restantes sí hay, pero suelen ser muy breves. Así la chria, la refutatio y la confirmatio tienen tres; el locus communis, cinco; la thesis, seis; la sententia, ocho; la descriptio, nueve; la ethopoeia, diez y la comparatio, quince. La mayoría están precedidos de las siglas V. g., uerbi gratia, y los cita textualmente, acompañados del autor y la obra, como en la sententia: V. g. Alium silere quod uoles, primus sile ${ }^{42}$, Sen. In Hippol. (DRD 1688, p. 5); o solo del autor, en el mismo ejercicio: V. g. Ovid. Donec eris felix, multos numerabis amicos; / Tempora si fuerint nubila, solus eris ${ }^{43}$ (DRD 1688, p. 6); o sin mencionar ninguna de las dos cosas, cuando se trata de ejemplos de tradición, como en el locus communis: $\mathrm{Si}$ in Caini parricidium, in Iudae auaritiam, [...] inueharis; eadem oratio in omnes uel parricidas, uel auaros $[\ldots](D R D 1688$, p. 8) o de carácter proverbial, como en la sententia: Nulla quam lingua pestis est deterior (DRD 1688, p. 6).

Algunas partes de los ejercicios, como la definición, tipología y capita, etc., van acompañados de pequeñas glosas explicativas que aclaran su significado, usos y aplicaciones, como por ejemplo en la chria, cuando explica el punto de amplificación 7. A testimonio, añade: 'rem propositam Scriptoris alicuius auctoritate confirmando' ( $D R D$ 1688, p. 5). Las aclaraciones, muy breves y las mínimas imprescindibles, tampoco son suyas, sino tomadas de sus fuentes, y se encuentran integradas en el texto. No hay en su manual escolios o explicaciones separadas como en otros humanistas ni tampoco guías de lectura

42 Sen. Phaedr. 876.

${ }^{43}$ OVID. Tr.1.9.5-6. El texto actual de Ovidio presenta la variante sospes en vez de felix que contiene el ejemplo y que es más coloquial. 
aclaratorias en los márgenes. En los capita amplificationis elimina los comentarios, cuando indica expresamente que los capita son los mismos que se han explicado ya en otro ejercicio anterior, como ocurre, por ejemplo, en el De confirmatione, en el que se limita a nombrarlos sin más: 1 . Ab encomio auctoris, 2. Ab expositione rei; 3. A manifesto...; etc. (DRD 1688, p. 8). En la sententia, solo los enumera, 'Sententia igitur dilatatur eisdem omnino capitibus, quibus Chriam dilatari diximus. Nimirum’ ( $D R D$ 1688, p. 6), y añade una aclaración de tipo léxico en tres de ellos: 1 . Ab encomio seu laude; 3. Ab Ratione, seu causa; 5. A similitudine seu Parabola (DRD 1688, p. 6). De esta forma evita las reiteraciones innecesarias y aligera el contenido teórico. No obstante, eso no le impide especificar los capita amplificationis, precedidos de un adverbio aseverativo, unas veces, nempe, como en la sententia, y, otras, nimirum, como en la confirmatio. Creemos que la enumeración no le parece superflua, porque se trata de los ejercicios iniciales, el III y el IV, pues, en los más avanzados como en el IX, la uituperatio, el X, la comparatio, y el XII, la descriptio, únicamente especifica con qué otro progymnasma comparten los capita sin nombrarlos. En los dos últimos, el XIII, la thesis, y el XIV, la legislatio, los enumera sin más explicaciones. Las glosas de los capita amplificationis suelen llevar los verbos en $2^{\text {a }}$ persona de futuro imperfecto de indicativo: celebrabis, conferes, ostendes (DRD 1688, p. 2) y también abundan en ellas los gerundios: exponendo describendoque, uestiendo, uersando ( $D R D$ 1688 , p. 2), porque utiliza una lengua muy coloquial y cercana para dirigirse a los alumnos. Un caso diferente, a pesar de ser el II progymnasma, es el De narratione. En este ejercicio, cuyo desarrollo incluye por primera y única vez en esta edición, solo enumera seguidos los capita amplificationis, sin número que los preceda, ni uso de cursiva ni explicación complementaria: 'Dilatatur Narratio, si circunstantiis amplificetur, a qua persona, $\sigma^{\circ}$ a quo tempore,... diligenter exponatur'. (DRD 1688, p. 3). Todo lo relativo a este ejercicio, como ya hemos señalado, solo aparece en esta edición, pues lo considera propio de un nivel más alto, el de la Retórica, a la que remite como en las otras ediciones.

Al final de algunos progymnasmata, locus communis, laus, ethopoeia, thesis y lex, Alcázar coloca un calderón ( $)$ para iniciar un párrafo en el que recoge advertencias y observaciones o para agregar algún dato más a tener en cuenta o que encuentra relevante. En el locus communis añade tres, el primero entre los capita amplificationis y los capita aduersus reum. Los otros dos al final, como en los demás ejercicios. El contenido de estas notas tampoco es 
original, incluso utiliza expresiones usuales en Pomey, así la que emplea en De laude: Haec rudi penicillo expressimus, de eodem argumento pro rei dignitate oportunius tractaturi Rhetor. Lib. I. cap. 10 (DRD 1668, p. 10). En Pomey 1661, p. 402: tametsi rudi penicillo delineatum.

Alcázar ha suprimido el ejemplo ${ }^{44}$ que en Aftonio seguía a la explicacion teórica de cada progymnasma. Pensamos que en esta obra al humanista solo le ha interesado resumir la teoría y que para la práctica recurriría a la Silua selectorum triplex ${ }^{45}$, obra en la que recopila un gran número de Progymnasmata del también jesuita Jacobo Pontano ${ }^{46}$.

Como se puede constatar por lo expuesto, no hay nada original en el contenido de los Progymnasmata de Alcázar ni en la preceptiva teórica de los ejercicios ni en los comentarios ni tampoco en las observaciones finales que añade mediante calderones. Ese no ha sido el propósito de su autor, como ya dejó bien patente desde la portada de la obra. Su valor reside en su acertada selección de las versiones latinas, la de Agricola partim, partim Catanaeo de Lorich y las de Bravo y Pomey, que bebieron de ella; en su capacidad para extraer lo esencial de la preceptiva y de sintetizarlo de forma clara y precisa; $y$, también, en su capacidad de escoger lo mejor de cada versión y de fusionarlo sin omitir los conocimientos básicos de la preceptiva retórica de los progymnasmata.Todo ello convirtió su obra en un manual muy útil para la docencia hasta la expulsión de los jesuitas en 1767. Alcázar había afirmado de sí mismo en el segundo prólogo de las ediciones de 1681, Architectus sum, y como tal reorganizó las obras de otros de la forma que consideró más adecuada para los fines docentes que se había propuesto.

${ }^{44}$ Solamente hay dos ejemplos en la laus. Sobre esto puede verse M.E. Cuyás de Torres, 2014, p. 158 , n. 21.

45 B. Alcázar, 1687a y 1687b. Puede verse el contenido de esta obra en M.E. Cuyás de Torres, 2018a, p. 275, n. 25 y F. Sánchez Ruiz, 1947-1948, p. 696.

${ }^{46}$ F. Sánchez Ruiz, 1947-1948, p. 699 recoge la opinión que tenía Alcázar sobre Pontano: Pontanus Jacobus Jesuíta (sic) Cuius Dialogi morati sunt atque eruditi, e Tullio, Terentio ac Plauto hausti. A Schor. Vir in humanioribus potissimum litteris exercitatíssimus (sic). Alegambe. Con esta última palabra parece indicar Alcázar que su fuente ha sido el historiador jesuita Felipe Alegambe (1592-1652), natural de Bruselas, que escribió un catálogo sobre escritores de la Compañía, Bibliotheca Scriptorum Societatis Iesu (1642). 


\section{5.- Conclusiones}

El estudio de los Progymnasmata Aphthonii de Bartolomé Alcázar aporta las siguientes conclusiones:

Alcázar compuso y publicó un breve manual con el único propósito de iniciar a los alumnos de forma sencilla en los preceptos retóricos básicos antes de que accedieran a los estudios de Retórica. Para ello se sirvió de la obra de Aftonio, cuyo éxito editorial desde sus primeras traducciones al latín no había tenido parangón con ningún otro texto dedicado a la docencia.

$\mathrm{Su}$ contenido se centra en la parte teórica de Aftonio, es decir, en las definiciones, tipología o clases, y puntos de argumentación de cada ejercicio, aunque en algunos progymnasmata, cuando lo considera pertinente, añade, como Aftonio, otras informaciones, por ejemplo, etimología en la chria y el locus communis, partes en la fabula y virtudes en la narratio. No presenta guías de lectura ni recoge los exempla o aplicación práctica de los ejercicios de Aftonio.

Los Progymnasmata Aphthonii de Alcázar, como anuncia desde la propia portada, "Opus ex Optimis Rhetoribus collectum", son una versión fusionada de otras anteriores. Él se ha limitado a organizar las versiones de la parte teórica de los autores escogidos, a darles una nueva estructura y a unificarlas, añadiéndoles, cuando los encuentra imprescindibles para una mejor comprensión del contenido, breves comentarios, extraídos de las mismas fuentes. Lo mismo cabe decir de las advertencias finales introducidas por calderones, que añade en algunos ejercicios.

Las fuentes de su obra son tres: Reinhard Lorich, Bartolomé Bravo y Francisco Pomey. La elección de sus fuentes la determina en cada momento la brevedad y claridad de las exposiciones de sus modelos. Guiado por estos criterios, en cada ejercicio sigue básicamente a uno de ellos, preferentemente a Pomey, pues su traducción latina destaca por estas cualidades. Este sistema de copia no impide que la versión elegida aparezca unificada y complementada con alguna de las otras dos o, incluso, con ambas y que casi siempre utilice, para mejorar y clarificar las partes más complejas, el texto o los escolios de Lorich, modelo también de Bravo y Pomey. También se ha evidenciado que hay pasajes, cuya única fuente es Lorich, así ocurre con el Prooemium y con la descriptio, en los que Alcázar entremezcla la traducción del texto de Aftonio de AgrícolaCataneo con los escolios del humanista alemán. 
En cuanto al uso de las fuentes, se ha demostrado que las copia literalmente, las simplifica o las adapta. Se ha mostrado que, en ocasiones, altera la posición de algunos términos, amplía otros - especialmente los verbos, a los que añade prefijos o implementa con algún complemento implícito en el contexto - y sustituye algunos por términos más usuales o comunes. También se ha subrayado que en su redacción recurre a menudo a expresiones coloquiales, como el uso de gerundios o del futuro para dirigirse a los alumnos y explicarles cómo deben aplicar los capita amplificationis en la realización de los ejercicios. Sus Progymnasmata son realmente un epítome de las versiones de los tres autores que él ha considerado los optimos rhetores e intérpretes de la obra original.

Se ha expuesto que la $D R D$ (1668) presenta dos novedades con respecto a las anteriores ediciones de los Progymnasmata: en ella desarrolla por primera y única vez la narratio ( $D R D$ 1688, pp. 3-4) e introduce algunas innovaciones en la descriptio ( $D R D$ 1688, pp. 13-14), al reagrupar la clasificación de sus otras ediciones según un nuevo orden alfabético-semántico más lógico y fácil de memorizar por los alumnos.

Por todo ello, se llega a la conclusión de que el manual no contiene ningún precepto retórico nuevo atribuible a Alcázar y que el mérito de la obra reside en la destreza didáctica de su autor, en la acertada elección de sus modelos, en su capacidad de síntesis para copiar inteligentemente lo esencial de la preceptiva retórica de los progymnasmata y, por último, en su gran habilidad para fusionarlos en un manual sencillo, muy breve y claro, “in breuissimam formam dilucidamque digestum”, que permite al alumno el aprendizaje gradual del discurso oratorio mediante el conocimiento elemental de los ejercicios preparatorios, por lo que su función pedagógica es encomiable y explican su éxito en la enseñanza jesuítica hasta que estos fueron expulsados de nuestro país.

Estas conclusiones nos llevan a afirmar que la finalidad principal de esta obra fue de carácter puramente didáctico: el aprendizaje teórico de la preceptiva retórica de forma clara, precisa y rápida, y que, aunque su difusión fue interrumpida en 1767 , hoy ha vuelto a ser estudiada y editada, porque su conocimiento resulta imprescindible por la claridad y precisión de sus exposiciones sobre los progymnasmata y porque proporciona una base rápida y útil para la iniciación en la Retórica. 


\section{6.- Referencias bibliográficas}

\section{Fuentes:}

B. Alcázar, 1681a, Silua selectorum tripartita ex probatissimis latini Sermonis authoribus collecta: Pars I Oratoria $\mathcal{E}^{2}$ Historica, Pars II Didactica, Pars III Poetica, Mantuae Carpetanorum, typis et sumptibus Io. Garciae Infançonis.

B. Alcázar, 1681b, De ratione dicendi. Opusculum triplex siue I. De Aphthonii sophistae progymnasmatis, II De conscribendis epistolis, III De rhetorica facultate libri quinque..., Mantuae Carpetanorum, typis $\sigma^{\circ}$ sumptibus Ioannis Garciae Infançonis.

B. Alcázar, 1687a, Silua selectorum triplex, pars prima generistarum, pars secunda praeteristarum. Ad usum nobilissimae Iuuentutis Madridiensis,..., Mantuae Carpetanorum, typis Ioannis Garciae Infançonis.

B. Alcázar, 1687b, Silua selectorum triplex, pars tertia Syntaxistarum. Ad usum nobilissimae Iuuentutis Madridiensis,..., Mantuae Carpetanorum, typis Antonii Roman.

B. Alcázar, 1688, De ratione dicendi. Opusculum triplex, Matriti, ex typographio Jo. Garciae Infançonis, <http://bdh-rd.bne.es/viewer.vm?id=0000089 610> [consultada 25/02/2020].

B. Alcázar, 1706, Sylua selectorum tripartita ex probatissimis Latini sermonis authoribus collecta: Pars I Oratoria $\mathcal{E}^{\circ}$ Historica, Pars II Poetica, Pars III Didactica, Mantuae Carpentanorum, Haeredes Antonii Roman.

B. Alcázar, 1710, Chrono-historia de la Compañía de Jesús en la provincia de Toledo y elogios de sus varones ilustres, fundadores, bienhechores, fautores, e hijos espirituales, Madrid, Juan García Infançon.

B. Bravo, 1591, Liber de conscribendis epistolis, ac de progymnasmatis, seu praeexercitationibus Oratoriis cum singulis tum cuiusque generis Epistolarum, tum singulorum progymnasmatum exemplaribus, Segouiae, Petrus Rhemensis.

B. Bravo, 1596, De arte oratoria ac de eiusdem exercendae ratione Tullianaque imitatione, uaria ad res adibita exemplorum copia libri quinque, Methimnae a Campo, excudebat Iacobus a Canto, <http://www.larramendi.es/es/ consulta/registro.do?id=27710 $>$ [consultada 25/02/2020]

M. López Muñoz (ed. y trad.) 2019, Bartolomé Alcázar. De ratione dicendi, Madrid, Clásicos Dykinson. 
R. Lorich, 1670, Aphthonii Progymnasmata, a Rodolpho Agricola partim, partim a Johanne Maria Catanaeo, Latinitate donata. Cum scholiis $R$. Lorichii et accesione noua uariationum quibus fabulae et chreiae tractandae sunt, Vesaliae, Andreas ab Hoogenhuysen.

M. Patillon (éd. y trad.), 2008, Corpus Rhetoricum. Anonyme Préambule à la Rhétorique. Aphthonios Progymnasmata en annexe Pseudo-Hermogène Progymnasmata, Paris, Les Belles Lettres.

F. Pomey, 1661, Candidatus Rhetoricae seu Aphthonii progymnasmata in meliorem formam usumque redacta, Lugduni, apud Antonium Molin, e regione Collegii, <urn:nbn:de:bvb:12-bsb10574438-6> [25/02/2020].

Ratio atque Institutio studiorum Societatis Iesu, 1599, Neapoli, ex Typographia Tarquinii Longi.

M. D. Reche Martínez, 1991, Teón, Hermógenes, Aftonio. Ejercicios de retórica, Madrid, Ed. Gredos.

\section{Estudios:}

T. Arcos Pereira - M.E. Cuyás de Torres, 2008, "Los Comentarios a los Progymnasmata de Aftonio de Francisco Escobar y Juan de Mal Lara: estudio preliminar", en J. M. Maestre Maestre, J. Pascual Barea, L. Charlo Brea (eds.), Humanismo y Pervivencia del Mundo Clásico IV. Homenaje al Profesor Antonio Prieto, Alcañiz-Madrid, t. I, pp. 651-662.

T. Arcos Pereira, 2017, "La versión latina de Johannes Maria Catanaeus de los Aphthonii Progymnasmata: La editio princeps de 1507 y la edición romana de 1517", RELat, 17, pp. 137-153.

T. Arcos Pereira, (en prensa), "El De Arte Rhetorica libri quinque de Dominique de Colonia”, en Europa Renascens. Latín y vernáculo en los Siglos de Oro, Jaén-Baeza.

M.E. Cuyás de Torres, 2014, “Aftonio en el Renacimiento: El comentario al lugar común de Lorich”, HL, 63, pp. 153-177.

M.E. Cuyás de Torres, 2016, "Las ediciones de los Progymnasmata Rhetorica de Antonio Llull”, Cuad. filol. clás. Estud. lat. 36(2), pp. 255-277.

M.E. Cuyás de Torres, 2018a, "Una nueva edición de los Aphthonii Sophistae Progymnasmata de Bartolomé Alcázar”, Ágora. Estudos Clássicos em Debate, 20, pp. 269-282.

M.E. Cuyás de Torres, 2018b, "Las ediciones de la Silua Selectorum Tripartita y del De Ratione Dicendi de Bartolomé Alcázar”, RELat, 18, pp. 255-282. 
M.D. García de Paso, 2015, “La sententia en Alardus Aemstelredamus”, en J. M. Maestre Maestre, S. I. Ramos Maldonado, M. A. Díaz Gito, Mª V. Pérez Custodio et alii (eds.), Humanismo y Pervivencia del Mundo Clásico, V. Homenaje al Profesor Juan Gil, t. 3, pp. 1257-1269.

M.D. García de Paso, 2018a, "De primis apud rhetorem exercitationibus praeceptiones Petri Mosellani: el primer manual de progymnasmata escrito en latín por un humanista”, RELaT, 18, pp. 159-179.

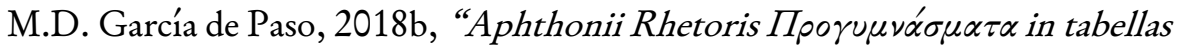
redacta et exemplis singulis illustrata de Matthaeus Bader: Un resumen de los Progymnasmata de Aftonio", Euphrosyne, 46, pp. 379-391.

E. Gil, 1992, El sistema educativo de la Compañía de Jesús: la "Ratio Studiorum", Madrid, UPCO.

F. Sánchez Ruiz, 1947-1948, “El humanista P. Bartolomé Alcázar de la Compañía de Jesús (1648-1721)”, en Anales de la Universidad de Murcia (1947-1948), Murcia, pp. 649-840. 